\title{
Analysis of the mainstream technology of mobile payment Weiwei Lang
}

Professional Department, Beijing Information Technology College,Beijing, China

langww@bitc.edu.cn

\begin{abstract}
Keywords: Mobile payment; NFC technology;infrared payment technology ; bluetooth payment technology; working principle
\end{abstract}

\begin{abstract}
With the development of mobile payment is more and more widely applied, as mobile payment technology mainstream NFC technology, has gradually been concerned because of its advantages compared with Bluetooth and infrared payment technology, is widely used in the field of mobile payment. This paper mainly carries on the elaboration from the development of NFC technology, working principle, characteristics and security aspects.
\end{abstract}

\section{Introduction}

Mobile payment, also known as mobile phone payment services, one way is to allow users to use the mobile terminal to the consumption of goods or services to account payment. Mobile payment includes remote payment and near-field payment. And NFC is the mainstream technology of near field pay.

\section{Brief introduction of NFC Technology}

NFC English full name Near Field Communication, the short distance wireless communication. It is sponsored by the Philips company, a wireless technology by Nokia, Sony and other famous manufacturers main push. Not long ago, by many companies, University and users set up a pan European Union, open architecture to the development of NFC, and to promote its application in mobile phone. NFC is composed of a non-contact radio frequency identification (RFID) and interconnection technology integration evolved, on a single chip with inductive card reader, induction card and point to point features, can be compatible with the equipment identification and data exchange in short distance. This technique was originally just a simple merging of RFID technology and network technology, it has evolved into a kind of short distance wireless communication technology, the development trend of quite quickly. Unlike RFID, NFC has a twoway connection and identify the characteristics, work in the $13.56 \mathrm{MHz}$ frequency range, about 10 $\mathrm{cm}$ distance effect. The NFC chip in the mobile phone, mobile phone can realize the micro electronic payment and read other NFC device or tag information. NFC short distance interaction greatly simplify the whole authentication process, make the electronic equipment room visit each other more direct, more secure and more clearly. Through the NFC, between the computer, mobile phone, digital camera, PDA and so on multiple devices can be very convenient for the wireless connection, and then to realize the data exchange and service. The transmission rate can be $106 \mathrm{kbit} / \mathrm{s}, 212 \mathrm{kbit} / \mathrm{s}, 424 \mathrm{kbit} / \mathrm{s}$, the future can be increased to more than $848 \mathrm{kbit} / \mathrm{s}$ [1].

The NFC terminal has three working modes: (1) the active mode, the NFC terminal as a reader, take the initiative to make their RF field to identify and read / write another NFC equipment; (2) the passive mode, the NFC terminal can be modeled by a card read / write, it is only in the RF field other equipment in the passive response; (3) the bidirectional mode, both sides actively send RF field to establish point-to-point communication. NFC is proposed for wireless communication between devices over a short range, can be used as a virtual connector, it can satisfy any of the two wireless device for data exchange between. It can also through Bluetooth initialization device and the original 802.11 wireless protocol, so that equipment can be farther in distance communication or in a more high rate data transmission. So in addition to the information transmission, the NFC 
device can be used as a security gateway in the network world, let users either at home or mobile, can store or receive all kinds of information at any time. As long as the two NFC devices together, they will automatically start the network communication function, the user need not be separately set installation procedure, thereby realizing the non-contact mobile payment, identification and other electronic purse and ID function. NFC technology in line with the international organization for standardization of ISO18092, ISO21481 standard, ISO14443 standard compatible wireless smart card, in line with European Computer Association of EMCA-340, 352 and 356 standard [2]. NFC compatibility contactless smart card field two strong -- FeliCa technology and MIFARE technology Philips Sony, the former smart card has deployed about 1200000000 piece based on, the latter has deployed about 170000000 tablets. This makes NFC technology fully has the characteristics of low power, low price of near distance, compatibility of the wireless interconnection equipment should be in the future, and make NFC a short distance wireless interconnection field of a highly competitive technology.

\section{The technical principle of NFC}

NFC device can exchange data in the active or passive mode. In passive mode, start the NFC communication equipment, also known as the NFC launch equipment (main equipment), provides the RF field in the whole communication process (RF-field). It can choose 106kbps, 212kbps or $424 \mathrm{kbps}$, where in a transmission speed, sends data to another device. Another device called the NFC target device (from the equipment), need not generate the RF field, while the load modulation (load modulation) technology, which can be the same speed returns data to launch equipment. This communication mechanism and based on ISO14443A, MIFARE and FeliCa contactless smart card compatible, therefore, NFC launch equipment in passive mode, you can use the same connection and initialization process testing contactless smart card or NFC target device, and link with the. The graph is NFC active communication mode.

In the active mode to send data to another device, each device must, must produce their RF field. As shown in Figure 1, initiating device and target device to generate their own RF field, for communication. This is the standard model of peer to peer network communication, can obtain very fast connection settings.

As shown in Figure 2, the mobile equipment to the main passive mode operation, can greatly reduce the power consumption, and prolong the service life of batteries. In an application session, NFC devices can switch their roles in between the initiating device and target device. Using this function, the battery low equipment may require the target device in passive mode, rather than initiating device. 
the initial order

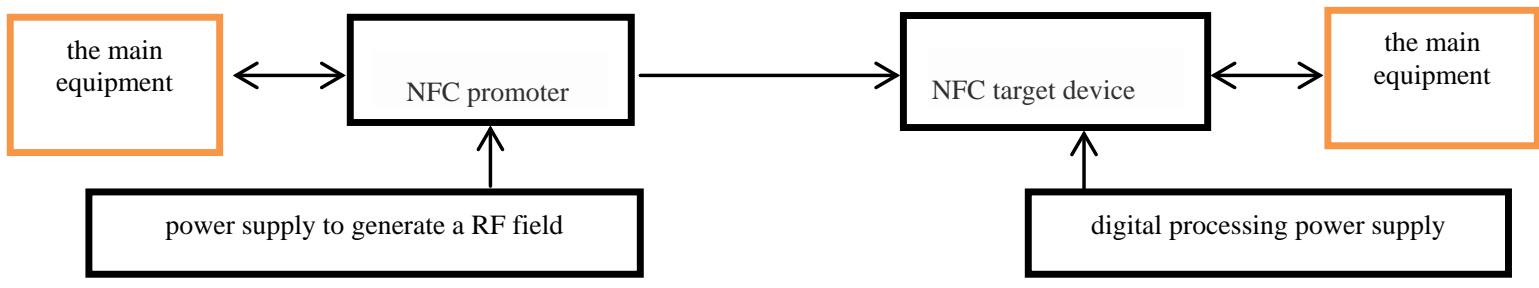

1. the initiator according to the selected speed begins to communication

answer

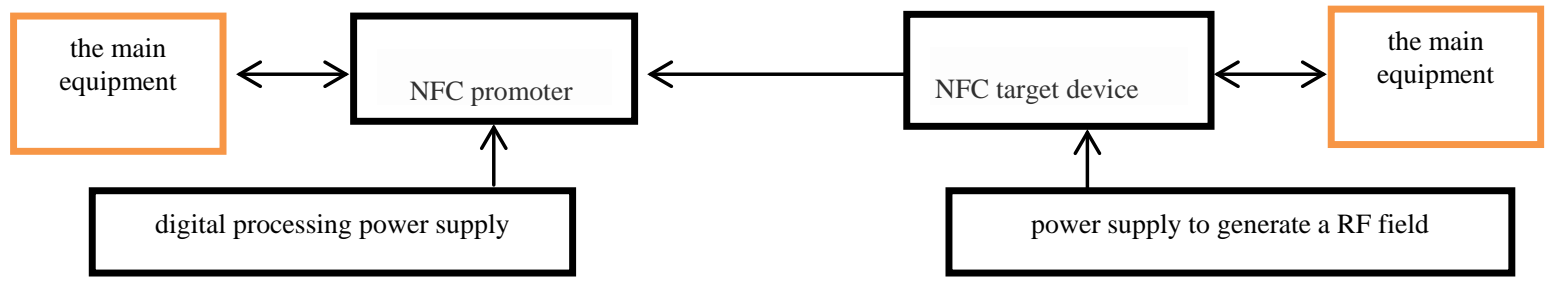

2. The target device according to the response speed of

Figure 1 NFC active communication mode

1. the initiator according to the selected speed begins to communication

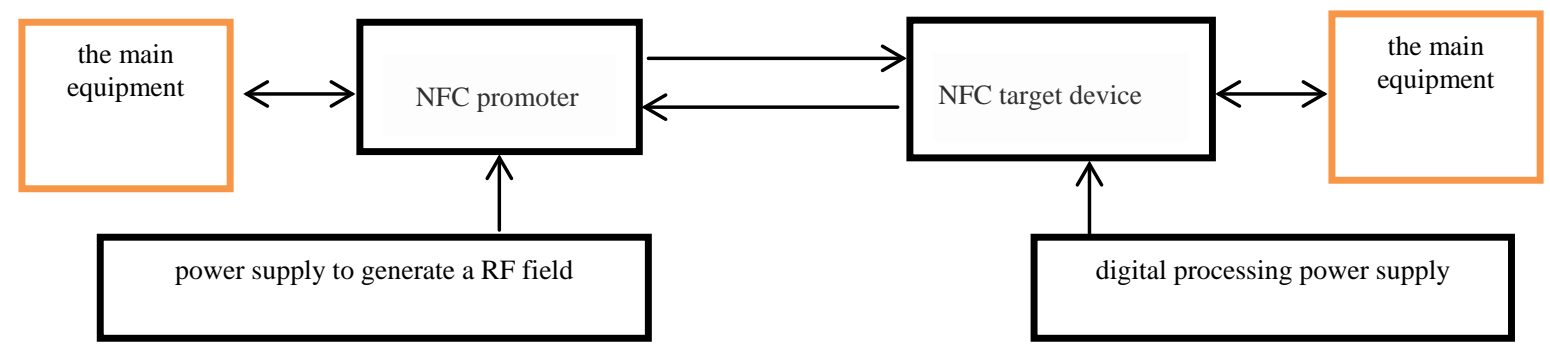

2. The target device according to the response speed of the

Figure 2 NFC passive communication mode

\section{The technical principle of NFC}

In the near field of payment function now there are mainly three kinds of technical means of payment including Bluetooth, infrared payment, NFC. Below we through the contrast analysis of the advantages and disadvantages of these three kinds of near-field payment technology.

\section{A. Bluetooth, infrared payment}

Bluetooth payment: Bluetooth is a short-range wireless network technology jointly launched by Ericsson, Nokia, Toshiba, IBM and Intel five world famous company in 1998, it works in the 2.4GHzISM band, the maximum transmission rate of 721Kbps. In 2001, Ericsson and EurocardAB began testing of Bluetooth Based Mobile Payment System in Sweden, mobile phone with Bluetooth function and pay Eurocard account of binding [3]. Features: the biggest obstacle of Bluetooth technology is too expensive, outstanding performance in the chip size and price is down, the anti- 
interference ability and the information security issues.

\section{B. Infrared payment}

Infrared payment: infrared payment is a mature and a contactless mobile payment technology. IrDA was founded in 1993, is a non-profit organization, committed to the establishment of the infrared wireless communication connection with international standards, has 160 members at present in the world. IrDA is using 980nm infrared band, receiving angle is 120 degrees, the transmission distance is directional $1 \mathrm{~m}$, speed of up to $16 \mathrm{Mbps}$. Features: IrDA's biggest problem lies in the existence of line of sight angle problem, that is to say between two IrDA ports of the equipment if the transmission data, the middle there can be no barrier. It is easy to realize between the two devices, but among a plurality of devices must adjust the position and angle of each other.

\section{NFC payment}

NFC is the most suitable technology for near-field payment technology.

NFC source on the RFID technology, but also different from RFID. NFC uses a two-way identification and connection, does not exist the master-slave relationship fixed communication parties, communication can be initiated by any of the NFC devices. Requirements of mobile within a 30 degree cone angle and not infrared communication equipment, and as a consumer payment technology, NFC infrared faster than, the operation is more simple. Compared with Bluetooth, NFC oriented close trading, applicable to exchange financial information or sensitive personal information and other important data; Bluetooth is suitable for long distance data communication, can make up for the deficiency of NFC communication distance. NFC and Bluetooth can complement each other, common existence. At the same time, the communication distance is very close also makes NFC have the security of natural. ZigBee is suitable for the field of industrial control [4] has a large number of wireless sensor and control operations, while the Wi-Fi is clearly more suitable for small office and home network. The cost of NFC, the low cost, Philips commitment, each chip cost around \$2. While the system cost Bluetooth, Wi-Fi and ZigBee are much higher than that of NFC.

Overall, as a kind of transaction oriented close distance wireless communication technology, NFC has obvious advantages, low power consumption, low cost, good safety, its rate can basically meet the demand of information exchange between devices, for video streaming applications requiring high bandwidth, can also with Bluetooth, Wi-Fi technologies, to provide automatic access function easily .

Table 1 is the comparison of the three .

\section{The security problems of NFC technology}

Although mobile payment, especially mobile phone payment means subject experts and businesses, banks, operators in favor, but it brings convenient. At the same time, people are worried about the safety of it how high.

In one survey, $40 \%$ of the domestic consumer lack of confidence in the security of mobile payment, only less than $15 \%$ of the mobile phone users complete trust in mobile payment, while $65 \%$ mobile phone users by mobile network to send their own refusal to credit card information. More than 90\% mobile phone users have received SMS fraud. Deputy general manager Cao Xiaoqing evaluation way China Financial Certification Center, "security issues will become one of the main factors restricting the development of mobile applications". In addition to worry about paying for security problems, worry about personal data leakage is the consensus of many users. 
Table 1 Comparison of the Bluetooth, Infrared payment, NFC

\begin{tabular}{|l|l|l|l|}
\hline Technical performance & Bluetooth & $\begin{array}{l}\text { Infrared } \\
\text { payment }\end{array}$ & NFC \\
\hline Dependent on the terminal & high & high & low \\
\hline Power consumption & large & large & small \\
\hline Simple operation & complicated & complicated & easy \\
\hline Confidentiality & medium & low & high \\
\hline Safety & high & low & medium \\
\hline The typical transmission distance & $<=10 \mathrm{~m}$ & $<=1 \mathrm{~m}$ & $<=0.1 \mathrm{~m}$ \\
\hline The transmission speed & $2.1 \mathrm{Mbps}$ & $115 \mathrm{kbps}$ & $106 / 212 / 424 \mathrm{Kbps}$ \\
\hline Isolated storage function & no & no & yes \\
\hline Setup time & $6 \mathrm{~s}$ & $0.5 \mathrm{~s}$ & $<0.1 \mathrm{~s}$ \\
\hline
\end{tabular}

In the process of mobile payment involved in payment participants include: consumer, merchant users, mobile operators, banks, the third party service providers. Consumer and business user is a system service object, provide network support for mobile operators, banks provide banking related services, third party service providers to provide payment services platform, combined by the parties to achieve business. Judging from the current situation, there are mainly three major factors influencing on the security issue of mobile payment by:

\section{Encryption and real-time problem}

Encryption and real-time problem is a major obstacle to the popularization of mobile phone payment, although the function of WAP mobile phone payments, mobile network can use encryption technology, relatively speaking, is not very effective to ensure safety. If the introduction of a text message confirming the realization of mobile phone payment double confirm mode, will be because of the problem of message relay, may cause message not arrive in time, affect the payment process.

\section{E. The lack of identification}

The lack of identification is to limit the application of mobile payment for second reasons. When the mobile phone calls just as tools, password protection is not very important. But as the payment tool, mobile information improves the mobile phone and other handheld terminal equipment problems important degree, loss, passwords are compromised, virus attack will cause a major loss.

\section{F. The lack of credit system}

The lack of credit system is the third largest cause of restrictions on the movement of information application. In mobile payment, some micro payment can be bound in the mobile phone, but the phone calls, malicious overdraft credit consciousness is very common, and the system is not perfect, but also restricts the popularization and promotion of mobile informatization. To solve this problem, mobile phone payment can be bundled with the mobile phone number, if the coordination management mechanism and technique control in place, carry on the safety of funds will be further improved. 


\section{Conclusion}

Mobile phone payment is paid an inevitable trend of development of the way, although the NFC mobile phone payment is not very perfect, but with the gradual solution of continuous progress and problems of the mobile payment technology, the development trend of mobile phone is still be a trend which cannot be halted, NFC technology will be applied more and more widely in the field of mobile phone payment .

\section{References}

[1]S.Ortiz.Jr.Is NearField Communication Close to Success[J]. IEEE Computer Society. March,200 6:18-20.

[2] Jiang Hua, sun Qiang. The short distance wireless communication technology standard analytic [J]. Nantong University School of electronics and information. Information technology and standardization of.2006 fifth: 26-30.

[3] C.Y. Leong, K. C. Ong, K. K. Tan, O.P. GAN.Near Field Communication and Bluetooth Brid [4]A.Zmijiewska.Evaluating Wireless Technologies in Mobile PaymentsA Customer Centric Approach[C].IEEE Proceedings of ICMB’05.Sydney:2005. 